\title{
Intracellular Penetration and Activity of DX-619 in Human Polymorphonuclear Leukocytes
}

\author{
Isabel García, ${ }^{1 *}$ Sofía Ballesta, ${ }^{1}$ Concepción Murillo, ${ }^{2}$ Evelio J. Perea, ${ }^{1,2}$ and Álvaro Pascual ${ }^{1,2}$ \\ Dept. of Microbiology, School of Medicine, University of Seville, ${ }^{1}$ and Dept. of Microbiology, University Hospital \\ Virgen Macarena, ${ }^{2}$ Seville, Spain
}

Received 5 April 2006/Returned for modification 7 May 2006/Accepted 2 July 2006

\begin{abstract}
The intracellular penetration and activity of DX-619 in human polymorphonuclear leukocytes have been evaluated. DX-619 reached intracellular concentrations 10 times higher than the extracellular concentrations reached. Uptake was rapid, reversible, nonsaturable, and affected by environmental temperature, some metabolic inhibitors, and a soluble membrane activator. DX-619 showed intracellular activity against Staphylococcus aureus.
\end{abstract}

The intracellular penetration and activities of antimicrobial agents are important considerations in the treatment of infections due to intracellular pathogens. Quinolones are able to penetrate and concentrate intracellularly, showing activities against intracellular pathogens $(1,6-8)$. DX-619 is a novel des-fluoro(6) quinolone that displays good activity against multiresistant gram-positive bacteria $(2,3)$. This quinolone also manifests an exceptionally low frequency of selection of resistant mutants $(16,17)$. One animal model shows that DX-619 could be a promising candidate for treating community-acquired respiratory infections (4). Its clinical role will be better appreciated when human pharmacokinetics data have been published. The purpose of this study was to evaluate the uptake of DX-619 by human polymorphonuclear leukocytes (PMNs). The mechanism involved in the penetration of this agent into human PMNs and its intracellular activity against Staphylococcus aureus were also evaluated.

The uptake of radiolabeled DX-619 (1.08 MBq/mg; Daiichi Pharmaceutical Co., Ltd., Tokyo, Japan) by human PMNs was determined by means of a velocity gradient centrifugation technique described by Klempner and Styrt (9). PMNs were incubated with different concentrations (1 to $40 \mu \mathrm{g} / \mathrm{ml})$ of DX-619. After different incubation times at $37^{\circ} \mathrm{C}$, cell-associated antimicrobial agent concentrations were calculated and expressed as ratios of cellular to extracellular concentration (C/E ratios) (11). The efflux of PMN-associated DX-619 was also studied. All assays were performed in duplicate with PMNs from four different donors. Data were expressed in terms of means \pm standard deviations. Differences among groups were compared by analysis of variance, with statistical significance being assessed at a $P$ value of $\leq 0.05$.

Figure 1 shows the kinetics of DX-619 uptake by and efflux from human PMNs. The uptake of DX-619 by human PMNs was a rapid process. DX-619 was highly concentrated in human PMNs, reaching intracellular concentrations 10 times higher than the extracellular concentrations reached after $20 \mathrm{~min}$ of incubation at $37^{\circ} \mathrm{C}$. $\mathrm{C} / \mathrm{E}$ ratios for $\mathrm{DX}-619$ were similar to

\footnotetext{
* Corresponding author. Mailing address: Department of Microbiology, School of Medicine, Avda, Sánchez Pizjuán s/n, Seville-41009, Spain. Phone: 34 954552862. Fax: 34 954377413. E-mail: igarcial@us.es.
}

those observed for moxifloxacin, gemifloxacin, sparfloxacin, and trovafloxacin and slightly higher than those observed for other quinolones, such as ofloxacin, levofloxacin, and ciprofloxacin $(5,6,12-15)$. The efflux of DX-619 from human PMNs was also a rapid process; when the extracellular antimicrobial was removed, PMN-associated DX-619 decreased 90\% after $10 \mathrm{~min}$ of incubation at $37^{\circ} \mathrm{C}$. The effect of different extracellular concentrations on the uptake of DX-619 by PMNs were also evaluated. Cell-associated DX-619 was not saturable at extracellular concentrations ranging from 1 to $40 \mu \mathrm{g} / \mathrm{ml}$ (data not shown).

Further studies to elucidate the mechanism of DX-619 uptake by PMNs were performed as described previously (12). The influences of cell viability, environmental temperature $\left(4^{\circ} \mathrm{C}\right.$ versus $37^{\circ} \mathrm{C}$ ), and external $\mathrm{pH}$ (5 to 8$)$ were evaluated. We also studied the effects of metabolic inhibitors (sodium fluoride, $1.5 \mathrm{mM}$; sodium cyanide, $1.5 \mathrm{mM}$; carbonyl cyanide $\mathrm{m}$ chlorophenylhydrazone, $0.015 \mathrm{mM}$; and 2,4-dinitrophenol, 0.1 $\mathrm{mM}$ [Sigma Chemical Co., St. Louis, Mo.]), membrane stimuli (phorbol myristate acetate [PMA] [200 nM; Sigma] and either opsonized zymosan [0.9 mg/liter; Sigma] or S. aureus ATCC 25923), and potential competitive substrates adenosine (1 $\mathrm{mM})$, L-lysine (1 $\mathrm{mM})$, and D-glucose (1 $\mathrm{mM})$ on DX-619 uptake by human PMNs.

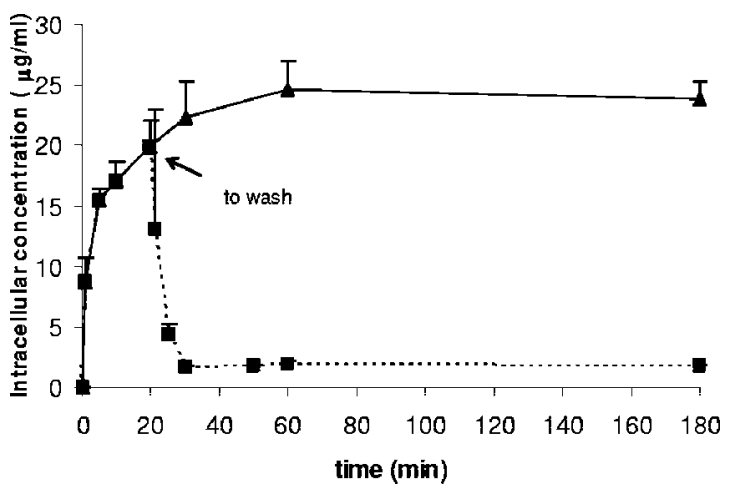

FIG. 1. DX-619 uptake by human PMNs and efflux of PMN-associated DX-619 after removal of the extracellular drug $(n=4)$. The extracellular concentration was $2 \mu \mathrm{g} / \mathrm{ml}$. Error bars indicate standard deviations. 


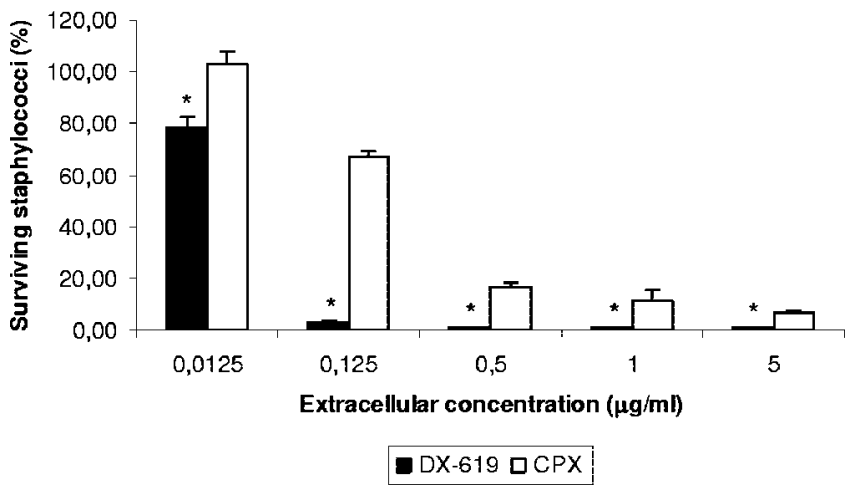

FIG. 2. Activities of DX-619 and ciprofloxacin against intracellular $S$. aureus in human PMNs $(n=4)$. Data are expressed as percentages of surviving bacteria after $3 \mathrm{~h}$ of incubation relative to controls without antimicrobial agents (means \pm standard deviations [error bars]). *, $P$ was $<0.05$ relative to ciprofloxacin.

DX-619 uptake was temperature dependent (C/E ratios, $1.2 \pm$ 0.4 versus $9.9 \pm 1.2)$ and significantly increased at acid $\mathrm{pH}$ (data not shown). Among the inhibitors evaluated, sodium cyanide and $m$-chlorophenylhydrazone significantly impaired intracellular penetration of DX-619 $(\mathrm{C} / \mathrm{E}$ ratios, $3.5 \pm 0.7$ and $5.4 \pm 1.1$, respectively; $\mathrm{C} / \mathrm{E}$ ratio for the control, $10.1 \pm 0.9)$. It is interesting that even an inhibitor of mitochondrial oxidative metabolism, such as sodium cyanide, decreased the uptake of DX-619 by PMNs since such cells have few mitochondria, as they are not dependent on oxidative metabolism for energy (12). This phenomenon could be related to secondary or minor activity of this inhibitor in other metabolic functions of phagocytes. However, preincubating the PMNs with sodium fluoride, an inhibitor of glycolysis (a major source of energy for the PMNs), did not affect their DX-619 uptake. These data point to the fact that a possible active mechanism could be partially involved in the uptake of this quinolone by human PMNs. Similar results were previously described for ofloxacin, levofloxacin, and moxifloxacin, all of which require an active process (13, 15). The uptake of other quinolones, such as sparfloxacin and trovafloxacin, by human PMNs was not affected by any of these parameters, and a typically passive mechanism is displayed $(5,14)$.

Particle phagocytosis or soluble membrane stimuli may modify the uptake of an antimicrobial agent by human PMNs. For DX-619, we observed a significant increase in its intracellular accumulation when human PMNs were stimulated with the soluble stimulus PMA (C/E ratios, $12.7 \pm 1.7$ versus $9.7 \pm$ 1.1). The phagocytosis of opsonized Staphylococcus aureus or zymosan, however, did not modify the uptake. This divergent effect could be related to the fact that PMN activation by opsonized particles differs from that by PMA (10). The findings presented suggest that DX-619 uptake is mediated by both active and passive diffusion across the cytoplasmic membrane of the PMN.

The intracellular activity of DX-619 compared with that of ciprofloxacin against $S$. aureus was evaluated in a 3-h assay using a previously described method (11). The MICs/minimal bactericidal concentrations of ciprofloxacin (Bayer AG, Leverkusen, Germany) and DX-619 against $S$. aureus ATCC 25923 were $0.25 / 0.5$ and $0.001 / 0.003 \mu \mathrm{g} / \mathrm{ml}$, respectively. Data were expressed as percentages of surviving staphylococci rela- tive to control levels (without antimicrobial agents) at $3 \mathrm{~h}$ (means \pm standard deviations). At extracellular concentrations of at least $0.0125 \mu \mathrm{g} / \mathrm{ml}, \mathrm{DX}-619$ showed significant intracellular activity against $S$. aureus. At all extracellular concentrations evaluated, the intracellular activity of DX-619 was significantly higher than that observed for ciprofloxacin (Fig. 2). The intracellular activity of DX-619 was dose dependent and greater than that of ciprofloxacin. This effect was partially due to the higher bactericidal activity of this antimicrobial against the strain evaluated.

In summary, DX-619 penetrates into human PMNs, reaching intracellular concentrations several times higher than the extracellular concentrations reached while remaining intracellularly active. A potentially active mechanism may be involved in the uptake of DX-619 by human PMNs. The high antimicrobial activity of this quinolone added to the properties observed in this study enlarges the potential use of DX-619.

\section{REFERENCES}

1. Baltch, A. L., L. H. Bopp, R. P. Smith, P. B. Michelsen, and W. J. Ritz. 2005 Antibacterial activities of gemifloxacin, levofloxacin, gatifloxacin, moxifloxacin and erythromycin against intracellular Legionella pneumophila and Legionella micdadei in human monocytes. J. Antimicrob. Chemother. 56:104-109.

2. Bogdanovich, T., D. Esel, L. M. Kelly, B. Bozdogan, K. Credito, G. Lin, K. Smith, L. M. Ednie, D. B. Hoellman, and P. C. Appelbaum. 2005. Antistaphylococcal activity of DX-619, a new des-F(6)-quinolone, compared to those of other agents. Antimicrob. Agents Chemother. 49:3325-3333.

3. Fujikawa, K., M. Chiba, M. Tanaka, and K. Sato. 2005. In vitro antibacterial activity of DX-619, a novel des-fluoro(6) quinolone. Antimicrob. Agents Chemother. 49:3040-3045

4. Fukuda, Y., K. Yanagihara, H. Ohno, Y. Higashiyama, Y. Miyazaki, K. Tsukamoto, Y. Hirakata, K. Tomono, Y. Mizuta, T. Tashiro, and S. Kohno. 2006. In vivo efficacies and pharmacokinetics of DX-619, a novel desfluoro(6) quinolone, against Streptococcus pneumoniae in a mouse lung infection model. Antimicrob. Agents Chemother. 50:121-125.

5. García, I., A. Pascual, M. C. Guzmán, and E. J. Perea. 1992. Uptake and intracellular activity of sparfloxacin in human polymorphonuclear leukocytes and tissue culture cells. Antimicrob. Agents Chemother. 36:1053-1056.

6. García, I., A. Pascual, S. Ballesta, P. Joyanes, and E. J. Perea. 2000. Intracellular penetration and activity of gemifloxacin in human polymorphonuclear leukocytes. Antimicrob. Agents Chemother. 44:3193-3195.

7. García, I., A. Pascual, S. Ballesta, and E. J. Perea. 2000. Uptake and intracellular activity of ofloxacin isomers in human phagocytic and nonphagocytic cells. Int. J. Antimicrob. Agents 15:201-205.

8. Jones, R. N. 2002. Microbiology of newer fluoroquinolones: focus on respiratory pathogens. Diagn. Microbiol. Infect. Dis. 44:213-220.

9. Klempner, M. S., and B. Styrt. 1981. Clindamycin uptake by human neutrophils. J. Infect. Dis. 144:472-475.

10. Maridonneau-Parini, I., S. M. Tringale, and A. I. Tauber. 1986. Identification of distinct activation pathways of the human neutrophil NADPH-oxidase. J. Immunol. 137:2925-2929.

11. Pascual, A., D. Tsakayama, J. Kovarik, G. Gekker, and P. K. Peterson. 1987. Uptake and activity of rifapentine in human peritoneal macrophages and polymorphonuclear leukocytes. Eur. J. Clin. Microbiol. Infect. Dis. 6:152-157.

12. Pascual, A., I. García, and E. J. Perea. 1989. Fluorometric measurement of ofloxacin uptake by human polymorphonuclear leukocytes. Antimicrob. Agents Chemother. 33:653-656.

13. Pascual, A., I. García, and E. J. Perea. 1990. Uptake and intracellular activity of an optically active ofloxacin isomer in human neutrophils and tissue culture cells. Antimicrob. Agents Chemother. 34:277-280.

14. Pascual, A., I. García, S. Ballesta, and E. J. Perea. 1997. Uptake and intracellular activity of trovafloxacin in human phagocytes and tissue-cultured epithelial cells. Antimicrob. Agents Chemother. 41:274-277.

15. Pascual, A., I. García, S. Ballesta, and E. J. Perea. 1999. Uptake and intracellular activity of moxifloxacin in human neutrophils and tissue-cultured epithelial cells. Antimicrob. Agents Chemother. 43:12-15.

16. Strahilevitz, J., Q. C. Truong-Bolduc, and D. C. Hooper. 2005. DX-619, a novel des-fluoro(6) quinolone manifesting low frequency of selection of resistant Staphylococcus aureus mutants: quinolone resistance beyond modification of type II topoisomerases. Antimicrob. Agents Chemother. 49:5051-5057.

17. Wickman, P. A., E. S. Moland, J. A. Black, and K. S. Thomson. 2006. In vitro activity of DX-619, a novel des-fluoro(6) quinolone, against a panel of Streptococcus pneumoniae mutants with characterized resistance mechanisms. Antimicrob. Agents Chemother. 50:796-798. 